\title{
Extra Axial Chordoma of the Distal Femoral Metaphysis: A Case Report
}

Long Nguyen

Cooper Medical School of Rowan University, nguyen17@rowan.edu Irene Vasko DO

Cooper University Hospital, Department of Diagnostic Radiology, Vasko-Irene@CooperHealth.edu

Gord G. Zhu DO

Cooper University Hospital, Department of Pathology, zhu-gordguo@CooperHealth.edu

Veniamin Barshay MD, MS, PhD

Cooper University Hospital, Department of Diagnostic Radiology, Barshay-Veniamin@CooperHealth.edu

Cooper Rowan Medical Journal: https://rdw.rowan.edu/crjcsm

Would you like to be a reviewer? Please fill in this short form to express your interest.

\section{Recommended Citation}

Nguyen, Long; Vasko, Irene DO; Zhu, Gord G. DO; and Barshay, Veniamin MD, MS, PhD (2020) "Extra Axial Chordoma of the Distal Femoral Metaphysis: A Case Report," Cooper Rowan Medical Journal: Vol. 2 : Iss. 1 , Article 4.

DOI: 10.31986/issn.2578-3343_vol2iss1.4

Available at: https://rdw.rowan.edu/crjcsm/vol2/iss1/4

\section{(c) (i)}

This work is licensed under a Creative Commons Attribution 4.0 License.

This Case Reports and Case Series is brought to you for free and open access by the Rowan University Journals at Rowan Digital Works. It has been accepted for inclusion in Cooper Rowan Medical Journal by an authorized editor of Rowan Digital Works. For more information, please contact brush@rowan.edu. 


\section{Extra Axial Chordoma of the Distal Femoral Metaphysis: A Case Report}




\title{
Extra Axial Chordoma of the Distal Femoral Metaphysis:
}

\section{A Case Report}

\section{Long Nguyen ${ }^{*}$, Irene Vasko, $\mathrm{DO}^{2}$, Gord G. Zhu, MD, MS, $\mathrm{PhD}^{3}$ \& Pauline Germaine, DO $^{3}$}

${ }^{1}$ Fourth-year MD Student Doctor, Cooper Medical School of Rowan University, 401 Broadway, Camden, 08103, NJ

${ }^{2}$ Fourth-year resident, Department of Radiology, Cooper University Hospital, 1 Cooper Plaza, Camden, 08103, New Jersey, United States

${ }^{3}$ Faculty, Department of Radiology, Cooper University Hospital, 1 Cooper Plaza, Camden, 08103, New Jersey, United States

*Corresponding author: nguyenl7@ rowan.edu (Long Nguyen)

\begin{abstract}
Background

Chordomas are malignant bone tumors that are derived from remnant embryonic tissue of the notochord and are typically found in the axial midline. When they are found outside of the axial skeleton, the diagnosis can be challenging and elusive. Often, they are overlooked on initial presentation in lieu of other more common lesions, including cartilage tumors (eg, enchondroma, chondrosarcoma, osteochondromatosis) due to their overlapping features.

Case Report

A 30-year-old female with a four-year history of intermittent left knee pain presented for initial evaluation. Physical exam of the knee was unremarkable except for moderate tenderness on palpation. Radiographs showed a lucent lesion with peripheral sclerosis, eccentrically located within the anteromedial femoral diaphysis. The patient was subsequently lost to follow-up.

She presented again two years later with similar symptoms. Her physical exam remained unchanged, and repeat radiographs showed interval growth. She underwent open biopsy of the left distal femur, with pathology revealing tumor consisting predominantly of epithelioid cells set in an abundant myxoid matrix. Immunohistology showed cells that stained strongly for EMA, CK19, and brachyury. These findings support the diagnosis of extra-axial chordoma.
\end{abstract}




\section{Discussion}

Extra-axial chordomas remain a challenging diagnosis for clinicians due to their extreme rarity and overlapping features with other more common lesions. They are often misdiagnosed as extraskeletal myxoid chondrosarcomas or myoepitheliomas. Our patient's insidious presentation and radiologic findings present a learning opportunity for clinicians to recognize extra-axial chordomas as a potential early diagnosis in someone presenting with a long history of intermittent and worsening knee pain.

\section{INTRODUCTION}

Chordomas are rare malignant bone tumors that are derived from remnant embryonic tissue of the notochord. These are low-grade neoplasms that exhibit slow growth but are locally aggressive and destructive, with a high potential for recurrence ${ }^{1-3}$. They have an incidence rate of 0.08 cases per 100,000 people and are almost exclusively located in the axial midline, with $32.8 \%$ of cases in the mobile spine, followed by the skull base (32\%) and the sacrococcygeal bones $(29 \%)^{4,5}$.

A small number of these tumors can also be found outside of the axial skeleton in the nasopharynx, posterior mediastinum, appendicular skeleton, and soft tissues ${ }^{6}$. These tumors are referred to as extra-axial chordomas, and they often share many identical features with their axial counterparts. Histologically, both types of tumors can have the characteristic physaliferous cells with a myxoid background.

Immunohistochemical studies classically show positive expression for brachyury, cytokeratins, and S-100 $4,5,7$. Brachyury, a transcription factor involved in the differentiation of the notochord, is a specific marker for notochordal tumors that allows for the differentiation of chordomas from other mimics, including metastatic mucinous adenocarcinomas and myoepithelial tumors.

Extra-axial chordomas can also share common features with cartilage tumors. As such, they are often overlooked on initial presentation until other more common tumors have been entertained. Here, we describe a rare case of a 30-year-old female who presented with an extra-axial chordoma in the left distal femur metaphysis after a long clinical course of left knee pain.

\section{CASE REPORT}

A 30-year-old female with a four-year history of worsening intermittent anteromedial left knee pain presented for an initial orthopedic consultation. On this first visit, the patient reported that the pain had become more constant, often worse following activity. Physical exam did not show any swelling, skin changes, erythema, or increased warmth. She had full left knee range of motion but was moderately tender on palpation. Her sensation, reflexes, and strength all remained within normal limits. She also denied any 
fever, chills, night sweats, or history of trauma.

Radiographs of the knee demonstrated a lucent lesion with peripheral sclerosis, eccentrically located within the anteromedial femoral diaphysis. The lesion was further characterized as expansile lesion (measuring $1.5 \times 1.5 \times 1.1 \mathrm{~cm}$ ) within the cortex of the anterior femur with $\mathrm{T} 2$ hyperintensity on magnetic resonance imaging (MRI). When compared to imaging performed two years prior, there was a mild interval increase in the size of the lesion, which previously measured $1.5 \times 0.9 \times 1.0 \mathrm{~cm}$. These findings had raised concerns for either an enchondroma or a periosteal chondroma. The patient was scheduled to undergo a curettage, biopsy, and possible allograft.

However, the patient later cancelled the surgery and was eventually lost to follow-up. She subsequently presented two years later with worsening intermittent left knee pain and difficulty climbing stairs. Her physical exam remained unchanged. Repeat radiographs of the left knee showed continued interval growth of the lesion, now measuring $1.3 \times 1.9 \times 2.6 \mathrm{~cm}$, but with otherwise similar imaging characteristics (Figures 1A and 1B). An MRI at the time confirmed an eccentric, cortically based lesion in the anteromedial distal femoral metaphysis with T2 hyperintensity (Figures 2A, 2B, and 2C). The patient did not present with any other symptoms that would warrant suspicion for metastasis or other new lesions, and further imaging was not recommended.

The patient underwent an open biopsy of the left distal femur with curettage of the bone lesion. The sample was sent to pathology with the final report revealing a diagnosis of extra-axial chordoma. The tumor consisted of predominantly epithelioid cells set in an abundant myxoid matrix (Figure 3). These cells contained hyperchromatic and pleomorphic nuclei with abundant eosinophilic and vacuolated cytoplasm. Immunohistochemical studies showed cells that stained strongly positive for EMA, CK19, SATB2 and brachyury. Focal expression of pancytokeratin (AE1/AE3), Cam5.2 and S100 were present as well. Ki67 proliferation index was $<5 \%$, consistent with a slow-growing tumor.

The pathology showed mild atypia in the stroma and an overall histology that was not overly malignant. The decision was made to meticulously cauterize the base of the lesion at this time and to exercise conservative management with routine clinical and radiographic surveillance. The patient presented to her first post-op visit ten days later. Examination of the extremity revealed a well-healed incision. The patient ambulated with normal gait using no assistive devices. She was recommended for physical therapy, which consisted of 2-3 planned sessions a week for a total of 8 weeks. Four-month post-op visit showed that the patient was still doing well. 

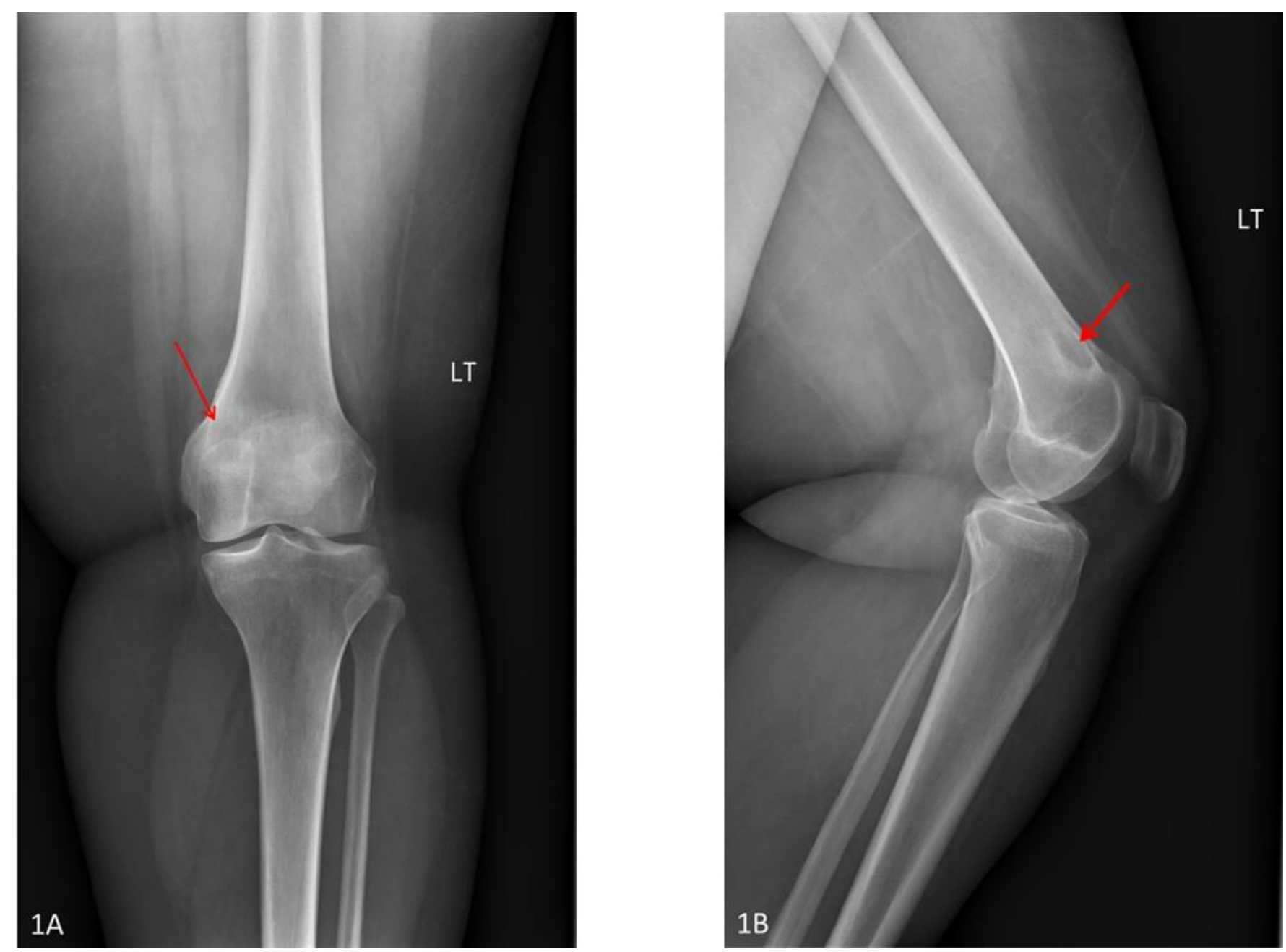

Figure 1 1A and 1B: Posterior-Anterior (PA) and Lateral radiographs of the left knee demonstrate a lucent lesion within the anteromedial distal femoral metaphysis with a sclerotic margin and narrow zone of transition 

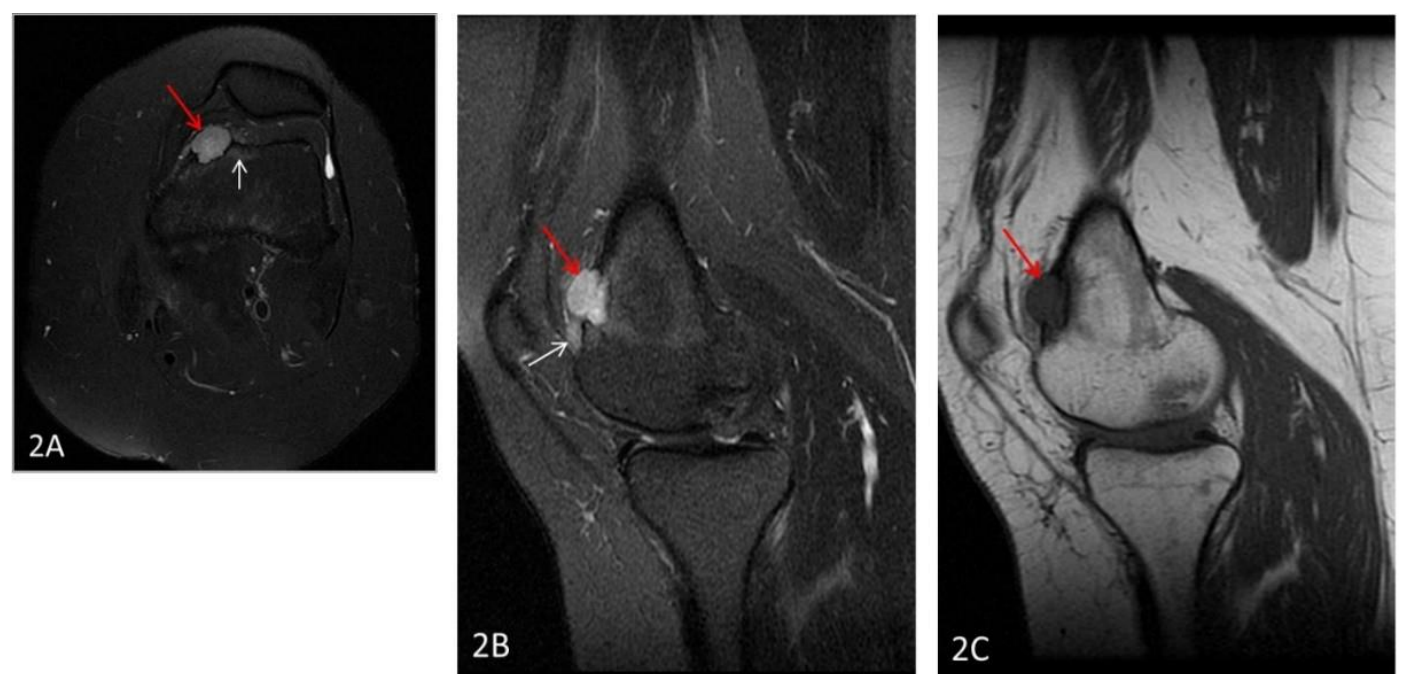

Figure 2 2A, 2B, 2C: Unenhanced magnetic resonance imaging of the left knee. Axial (Figure 2A) and sagittal (Figure 2B) T2 fat saturated images as well as a sagittal T1 (Figure 2C) image demonstrate an eccentric, cortically based soft tissue lesion (red arrow) with $\mathrm{T} 2$ hyperintensity and $\mathrm{T} 1$ hypointensity arising from the anteromedial distal femoral metaphysis. Minimal adjacent bone marrow and soft tissue edema are seen (white arrow). 

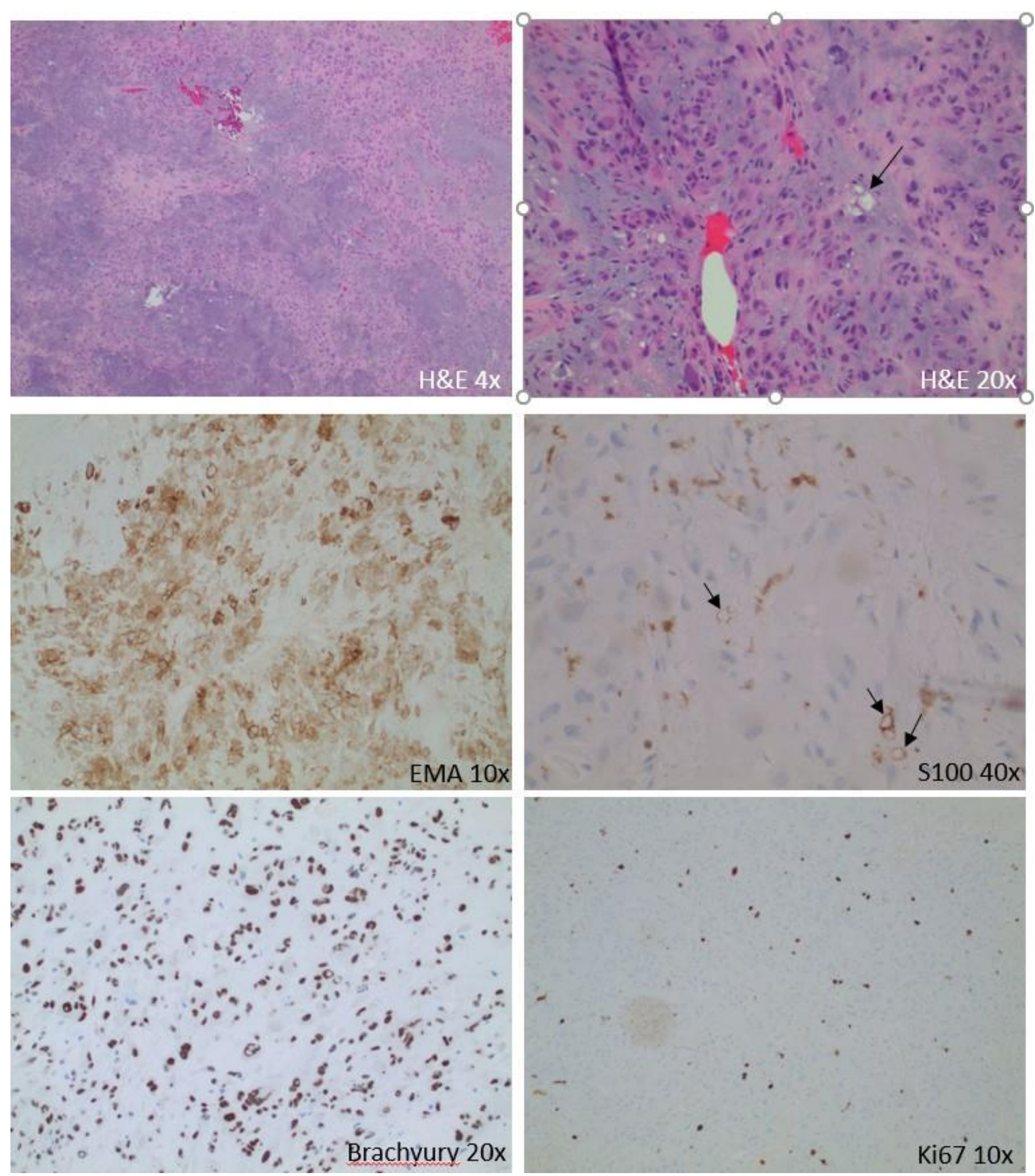

Figure 3 H\&E stains of the patient's biopsy sample, showing clusters and cords of pleomorphic epithelioid tumor cells set in an abundant myxoid matrix. On high power, tumor cells feature hyperchromatic and pleomorphic nuclei and abundant eosinophilic to vacuolated cytoplasm (arrow pointing to a physaliferous cell). The tumor cells are strongly positive for EMA and brachyury and variably positive for S100 (which highlights cytoplasmic vacuoles too, arrows). Ki67 proliferation index is $<5 \%$, consistent with a slow-growing tumor. The tumor cells are also positive for CK19, cytoplasmic SATB2, variably positive for PanCK (AE1/AE3), Cam5.2, while negative for CD31, CD34,

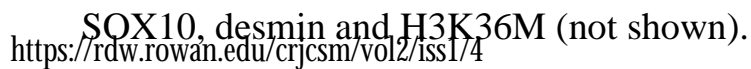




\section{DISCUSSION}

Extra-axial chordomas are extremely rare neoplasms that continue to be a challenging diagnosis for clinicians. For one, they are slow-growing tumors, and patients usually present after a long clinical course, often in their fourth to seventh decade ${ }^{8}$. The most common presentation is a slowly enlarging mass with localized pain ${ }^{9}$. Moreover, the findings of extra-axial chordomas often resemble those of well-differentiated cartilage tumors, such as an enchondroma, chondrosarcoma, or even synovial osteochondromatosis $^{5}$. Their similarities can be attributed to their common notochord origin. However, cartilage tumors are by far much more common, and their diagnoses are more likely to be considered first, as was the case with our patient ${ }^{10}$.

Imaging is the next step in the clinical assessment of these tumors, even though radiological findings often show much overlap between extra-axial and axial chordomas ${ }^{5}$. Radiographs demonstrate that chordomas are lytic and well-defined lesions with sclerotic margins ${ }^{4,7,11}$. There may also be an associated soft tissue mass and cortical permeation. On further evaluation with CT, chordomas classically are seen as circumscribed, hypoattenuating, expansile soft tissue masses with surrounding sclerosis or bone destruction $^{12}$. Calcifications may be present, and associated soft tissue components show moderate to significant enhancement on contrast enhanced $\mathrm{CT}^{2}$. MRI, however, is superior for tumor characterization and evaluation of disease extent. On T1-weighted sequences, chordomas demonstrate variable signal intensity, with most cases exhibiting low-to-moderate signal ${ }^{4}$. Small foci of hyper-intensity may be present, indicating underlying mucus or hemorrhage. On T2-weighted imaging, chordomas are classically hyperintense with possible heterogenous areas of hypointensity secondary to mucus or hemorrhage $e^{4,5,12}$. Enhancement is seen on post-contrast imaging. Unfortunately, these imaging features are non-specific and are similarly seen with cartilaginous tumors, making the diagnosis by radiologic findings alone difficult. Final diagnosis relies on careful histologic assessment assisted with the use of an array of immunohistochemical stains. Clinicians can use these markers in combination with radiographic evidence to diagnose chordoma with high confidence. Nuclear expression of the brachyury transcription factor is highly characteristic of chordomas ${ }^{11,13}$. Brachyury is a member of the T-box proteins that is highly sensitive and specific for early notochord differentiation ${ }^{5}$. Clinicians can use this finding to help distinguish skeletal and soft tissue extra-axial chordomas from other mimics and to establish a diagnosis. Other important positive markers include cytokeratins AE1/AE3, vimentin, S-100 protein, and epithelial membrane antigen (EMA), which can also add another degree of confidence in ruling out cartilage tumors. $^{11,12}$.

There is also the very rare challenge of differentiating extra-axial chordomas and metastasis from axial chordomas. While chordomas are known for their local aggressiveness and recurrence, metastatic disease 
has been reported in up to $10 \%$ of cases, with the most common site being the lungs ${ }^{9}$. There is some evidence in the literature that FDG PET/CT can help make the distinction between extra-axial versus metastatic axial disease. It has been reported that FDG PET/CT usually demonstrates increased uptake of FDG in axial chordomas ${ }^{2,8}$.

Extra-axial chordomas account for $6 \%$ of all chordomas, and their extreme rarity makes it a challenging diagnosis for clinicians and radiologists ${ }^{1}$. They can present atypically and in an indolent manner and are often misdiagnosed as chondrosarcomas or myoepitheliomas. To date, there have been 28 cases of extra-axial chordoma reported in the literature, but only two other cases of extra-axial chordoma of the femur ${ }^{4}$. Our presented case adds to the knowledge base of extra-axial chordomas, with emphasis on their histology, immuno-histochemical staining patterns as well as radiologic features. Clinicians can be better equipped to recognize extra-axial chordomas as a potential early diagnosis in the context of someone presenting with a long history of intermittent and worsening knee pain.

\section{REFERENCES}

1. Ueda T. Extra-Axial Chordoma of the Gingiva. Auris Nasus Larynx. 2019;.

2. Park S Y.Extra-Axial Chordoma Presenting as a Lung Mass. Respiration. 2009;77:219-242.

3. Difrancesco L, Davanzo C, Temple W. Extra Axial Chordoma. Arch Patho Lab Med. 2006;130:1871-74.

4. Righi A. Extra-Axial Chordoma: A Clinicopathologic Analysis of Six Cases. Virchows Arch. 2018;472:1015-1035.

5. Neumann J. Intra-Articular Extra-Axial Chordoma of the Wrist: A Case Report with Review ofthe Current Literature. Skeletal Radiol. 2019;

6. Lv G H. Clinicopathological and Prognostic Characteristics in Extra-Axial Chordomas: An Integrative Analysis of 86 Cases and Comparison with Axial Chordomas. Neurosurgery. 2019;85:527-569.

7. Huang J. Proximal Tibial Extra-Axial Chordoma Masquerading as Renal Cell CarcinomaMetastasis. Skeletal Radiol. 2017;46:1567-73.

8. Tsukamoto S. Parosteal Extra-Axial Chordoma of the Second Metacarpal Bone: A Case Report with Literature Review. Skeletal Radiol. 2018;47:579-85.

9. Evans S. Extra-Axial Chordomas. Ann R Coll Surg Engl. 2016;98:324-332. 
10. Muneer Mohammed, Badran Saif, Al-Hetmi Talal. A Rare Presentation of Axial Chordoma and the Approach to Management. American Journal of Case Reports. 2019;20:773-775.

11. Lantos J E. Recurrent Skeletal Extra-Axial Chordoma Confirmed with Brachyury: Imaging Features and Review of the Literature. Skeletal Radiol. 2013;42:1451-1460.

12. Chen Q Q. Chordoma Located in the Jugular Foramen: Case Report. Medicine (Baltimore). 2019;98.

13. Miettinen M. Nuclear Brachyury Expression Is Consistent in Chordoma, Common in Germ Cell Tumors and Small Cell Carcinomas, and Rare in Other Carcinomas and Sarcomas: An Immunohistochemical Study of 5229 Cases. Am J Surg Pathol. 2015;39:1305-1317.

14. Almefty Kaith, Pravdenkova Svetlana, Colli Benedicto O., Al-Mefty Ossama, Gokden Murat. Chordoma and chondrosarcoma: Similar, but quite different, skull base tumors. Cancer. 2007;110(11):2467-2477.

15. Noor Arish, Bindal Poorva, Ramirez Miguel, Vredenburgh James. Chordoma: A Case Report and Review of Literature. American Journal of Case Reports. 2020;21. 